\title{
Development of fever following first administration of zoledronate as a prognostic factor in advanced non-small cell lung cancer patients with bone metastases
}

\author{
HIROKI IZUMI $^{1}$, TADASHI IGISHI ${ }^{1}$, AKIRA YAMASAKI ${ }^{1}$, KENNICHI TAKEDA $^{1}$, MASAAKI YANAI ${ }^{1}$, \\ NATSUMI TANAKA ${ }^{1}$, TOMOHIRO SAKAMOTO ${ }^{1}$, SHIZUKA NISHII-ITO ${ }^{1}$, HIROKAZU TOUGE ${ }^{1}$, \\ MASAHIRO KODANI $^{1}$, SHINGO MATSUMOTO ${ }^{1}$, YUJI KAWASAKI $^{2}$ and EIJI SHIMIZU ${ }^{1}$ \\ ${ }^{1}$ Division of Medical Oncology and Molecular Respirology, Faculty of Medicine, Tottori University, Yonago, \\ Tottori 683-8504; ${ }^{2}$ Department of Respiratory Medicine, Tsuyama First Hospital, Nakashima, Tsuyama 708-0871, Japan
}

Received June 7, 2017; Accepted September 28, 2017

DOI: $10.3892 / \mathrm{mco} .2017 .1447$

\begin{abstract}
Nitrogen-containing bisphosphonates (N-BPs), which are usually used for the treatment of advanced cancer with bone metastasis, occasionally cause fever following the first administration. However, it is unclear as to how the development of fever following the first administration of N-BP is associated with clinical outcome. The aim of the present study was to determine the prognostic value of the development of fever following the first administration of N-BP in advanced non-small cell lung cancer patients with bone metastases. The present study reviewed the data of 46 patients with advanced non-small cell lung cancer who were administered zoledronate (ZOL), an N-BP, for bone metastasis, between March 2009 and March 2011 in the Department of Medical Respirology at Tottori University Hospital. Clinicopathological factors were evaluated using univariate and multivariate analyses, and these factors were compared between the fever and non-fever groups. Of the 46 patients, 15 (32.6\%) developed fever following the first administration of ZOL. No significant differences were observed in the clinicopathological characteristics between the two groups. The overall survival in the fever group was significantly longer compared with the non-fever group (median survival time: 33.4 vs. 15.7 months, $\mathrm{P}=0.04$ ), and the development of fever following
\end{abstract}

Correspondence to: Dr Hiroki Izumi, Division of Medical Oncology and Molecular Respirology, Faculty of Medicine, Tottori University, 36-1 Nishi-machi, Yonago, Tottori 683-8504, Japan

E-mail: h.izumi@med.tottori-u.ac.jp

Abbreviations: CI, confidence interval; ECOG-PS, Eastern Cooperative Oncology Group-performance status; EGFR, epidermal growth factor receptor; HR, hazard ratio; N-BPs, nitrogen-containing bisphosphonates; Sq, squamous cell carcinomas; NSCLC, non-small cell lung cancer; OS, overall survival; QOL, quality of life; SRE, skeletal-related events; ZOL, zoledronate

Key words: nitrogen-containing bisphosphonates, survival time, prognosis, prognostic indicator, non-small cell lung cancer the first ZOL administration was independently associated with longer overall survival. The development of fever following the first ZOL administration was an independent prognostic factor in advanced non-small cell lung cancer patients with bone metastases. Thus, ZOL-associated fever may be a predictive factor for an undefined, survival-promoting effect of ZOL.

\section{Introduction}

Lung cancer is the leading cause of cancer-related death worldwide (1), and bone metastases occur in $20-30 \%$ of patients with lung cancer (2). Bone metastases reduce the quality of life (QOL) of cancer patients by causing pain or skeletal-related events (SREs) such as pathological fractures, spinal cord compression, and hypercalcemia. Nitrogen containing bisphosphonates (N-BPs) are widely used to prevent SREs in cancer patients with bone metastases $(3,4)$. Preclinical studies have demonstrated the in vitro anticancer activity of N-BPs in multiple myeloma (5). In particular, the N-BP zoledronate (ZOL) has demonstrated survival benefits in premenopausal women with early-stage breast cancer and in previously untreated patients with multiple myeloma $(6,7)$. However, the survival benefit of ZOL in patients with cancer, including lung cancer, remains unclear.

N-BPs inhibit osteoclastic bone resorption by blocking farnesyl-pyrophosphate-synthase, an enzyme in the mevalonate pathway. The first administration of intravenous N-BPs is occasionally associated with the development of fever, which is due to the activation of $\gamma \delta \mathrm{T}$ cells, resulting in the release of interferon- $\gamma$, interleukin- 6 , and tumor necrosis factor- $\alpha(8,9)$. $\gamma \delta \mathrm{T}$ cells, a small subset of T lymphocytes, play an active role in immunosurveillance against tumors as well as infections, as components of innate immunity (10).

Therefore, we hypothesized that the development of fever after the first administration of N-BPs would be associated with the survival of patients with cancer. The aim of this retrospective study was to estimate the prognostic value of fever development after the first ZOL administration (ZOL-related fever) in advanced or recurrent non-small cell lung cancer (NSCLC) patients with bone metastases. 


\section{Patients and methods}

Materials. We retrospectively reviewed the data of patients who received a diagnosis of advanced or recurrent NSCLC between March 2009 and March 2011 at Tottori University Hospital in Japan and who received ZOL therapy for bone metastases. This retrospective study was approved by our institutional human research committee. The onset rate of ZOL-related fever is known to peak within the first 2 days after ZOL infusion and is short lived (11). Therefore, ZOL-related fever was defined as fever $\left(>37.5^{\circ} \mathrm{C}\right)$ that occurred within 2 days after the first ZOL administration and that did not persist for $>2$ days. Fever treated with antibiotics was not regarded as ZOL-related fever.

Methods and statistical analysis. The exclusion criteria included an Eastern Cooperative Oncology Group (ECOG) performance status (PS) score of 2-4 at diagnosis, and no data concerning fever after the treatment with $\mathrm{ZOL}$, even if records of ZOL administration were available. We collected clinicopathological data such as age, sex, smoking history, ECOG-PS, disease stage, histological type, epidermal growth factor receptor $(E G F R)$ mutation status, the time from the first $\mathrm{ZOL}$ administration to first SRE, and overall survival (OS) from the time of diagnosis of advanced disease or recurrence. The characteristics of patients with ZOL-related fever (fever group) were compared with those of patients without ZOL-related fever (non-fever group) by using the Mann-Whitney test and Fisher's exact test for numerical and categorized data, respectively. The OS and the time to first SRE were assessed using the Kaplan-Meier method and compared using the log-rank test. Multivariate testing was performed using Cox regression analysis with adjustments for factors that tended to be associated with OS $(\mathrm{P}<0.3)$, such as smoking history, histological type, EGFR mutation status, PS, and ZOL-related fever.

$\mathrm{P}<0.05$ was considered statistically significant. Statistical analyses were performed using PASW Statistics 19 (IBM SPSS Statistics, Somers, NY, USA).

\section{Results}

A total of 62 patients with advanced or recurrent NSCLC who were administered ZOL therapy were screened. Of these, 16 patients were excluded because of the lack of data concerning ZOL-related fever, and 46 patients were included in this retrospective study. The median follow-up time of patients was 15.7 months (range, 4.2-107.3 months). The patient characteristics for all 46 patients are listed in Table I. The age of patients ranged from 47 to 86 years (median age, 65 years), with $30(63.2 \%)$ men. Of the 46 patients, $37(80.4 \%)$ had non-squamous cell carcinomas (non-Sq) (adenocarcinoma; $\mathrm{n}=36$, large cell carcinoma; $\mathrm{n}=1$ ), and 6 had $\mathrm{Sq}$. Thirty-seven (80.4\%) patients had stage IV disease, and 7 (15.2\%) had stage IIIB disease. The remaining $2(4.3 \%)$ patients had recurrent disease after surgery. EGFR mutation status was tested in 39 of the 46 patients and was positive in 16 (34.8\%) patients. Seven patients had unknown EGFR mutation status, and all of these 7 patients were diagnosed with $\mathrm{Sq}(\mathrm{n}=6)$ or large cell carcinoma $(\mathrm{n}=1)$. All 16 patients harboring EGFR mutations received EGFR-tyrosine kinase inhibitors at least once. Twenty-nine patients $(80.5 \%)$ were former or current smokers.

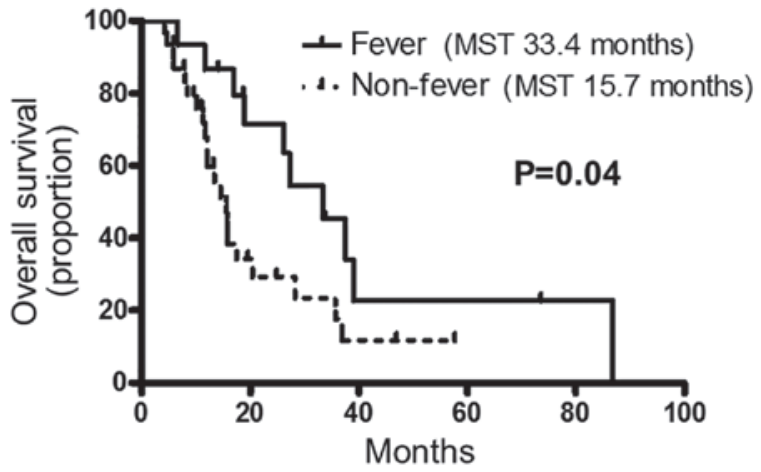

Figure 1. Kaplan-Meier curves of overall survival in the fever $(n=15)$ and non-fever groups $(\mathrm{n}=31)$. MST, median survival time.

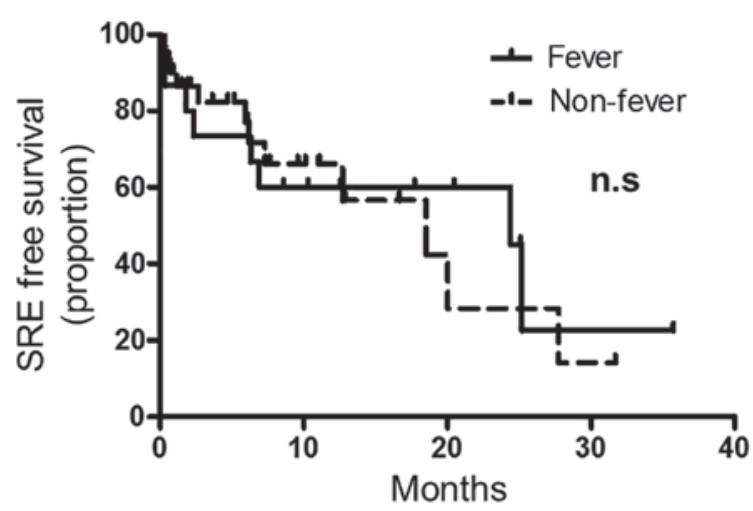

Figure 2. Kaplan-Meier curves of the time to first skeletal-related events in the fever $(n=15)$ and non-fever groups $(n=31)$. n.s., not significant

Patient characteristics including age, sex, EGFR mutation status, ECOG-PS, and smoking history were compared between the two groups (Table I). Compared with patients in the non-fever group, the patients in the fever group tended to be younger (median age, 62 vs. 66 years) and female (53.3 vs. $29.0 \%$ ), with a higher prevalence of ECOG-PS score 0 (33.3 vs. $16.1 \%$ ), but these differences were not significant.

Of the 46 patients, 15 (32.6\%) experienced ZOL-related fever after the first ZOL administration. To elucidate whether ZOL-related fever was associated with clinical outcome, we compared OS and time to the first SRE between the two groups. As shown in Fig. 1, the OS of fever group was significantly longer than that of the non-fever group (median survival time: 33.4 vs. 15.7 months, $\mathrm{P}=0.04)$ with a hazard ratio (HR) [95\% confidence interval (CI)] of $0.46(0.22-0.97)$. In addition, the median time to first SRE was 24.4 months in the fever group and 18.5 months in the non-fever group, with no significant difference between the two groups [HR $(95 \%$ CI) 1.3 (0.91-1.73), $\mathrm{P}=0.88$ ] (Fig. 2).

Positive EGFR mutation status [HR (95\% CI) 0.41 (0.20-0.86), $\mathrm{P}=0.016]$, non-Sq histology [HR (95\% CI) 0.24 (0.07-0.80), $\mathrm{P}=0.02]$, no smoking history [HR $(95 \% \mathrm{CI}) 0.37$ (0.20-0.80), $\mathrm{P}=0.001]$, and female sex [HR (95\% CI) 0.46 (0.22-0.95), $\mathrm{P}=0.036]$ were significantly associated with longer OS (Table II). In addition, multivariate analysis performed to test whether ZOL-related fever was an independent prognostic factor revealed that the presence of ZOL-related fever [HR (95\% CI) 0.37 (0.16-0.83), $\mathrm{P}=0.02$ ] and the absence of smoking 
Table I. Patient characteristics.

\begin{tabular}{|c|c|c|c|c|}
\hline Characteristics & Total & Fever & Non-fever & P-value \\
\hline No. of patients & 46 & 15 & 31 & \\
\hline Age in years, median (range) & $65(47-86)$ & $62(47-78)$ & $66(48-86)$ & 0.13 \\
\hline Sex & & & & 0.33 \\
\hline Female & $16(34.8 \%)$ & $7(46.7 \%)$ & $9(29.0 \%)$ & \\
\hline Male & $30(63.2 \%)$ & $8(53.3 \%)$ & $22(71.0 \%)$ & \\
\hline Disease stage & & & & 0.69 \\
\hline IIIB & $7(15.2 \%)$ & $3(20 \%)$ & $4(12.9 \%)$ & \\
\hline IV & $37(80.4 \%)$ & $11(73.3 \%)$ & $26(83.9 \%)$ & \\
\hline Recurrent & $2(4.3 \%)$ & $1(6.7 \%)$ & $1(3.2 \%)$ & \\
\hline Histological type & & & & 0.45 \\
\hline Squamous cell carcinoma & $9(19.6 \%)$ & $4(26.7 \%)$ & $5(16.1 \%)$ & \\
\hline Non-squamous cell carcinoma & $37(80.4 \%)$ & $11(73.3 \%)$ & $26(83.9 \%)$ & \\
\hline EGFR mutation status & & & & 0.74 \\
\hline Positive & $16(34.8 \%)$ & $6(40 \%)$ & $10(32.2 \%)$ & \\
\hline Negative or unknown & $30(65.2 \%)$ & $9(60 \%)$ & $21(67.8 \%)$ & \\
\hline ECOG-PS score & & & & 0.26 \\
\hline 0 & $17(37.0 \%)$ & $5(33.3 \%)$ & $5(16.1 \%)$ & \\
\hline 1 & $29(63.0 \%)$ & $10(66.7 \%)$ & $26(83.9 \%)$ & \\
\hline Smoking history & & & & 0.52 \\
\hline Never smoker & $10(21.7 \%)$ & $7(46.7 \%)$ & $10(32.2 \%)$ & \\
\hline Former or current smoker & $36(78.3 \%)$ & $8(53.3 \%)$ & $21(67.8 \%)$ & \\
\hline
\end{tabular}

ECOG-PS, Eastern Cooperative Oncology Group-performance status; EGFR, epidermal growth factor receptor.

Table II. Univariate and multivariate analyses of overall survival.

\begin{tabular}{|c|c|c|c|c|c|c|}
\hline \multirow[b]{2}{*}{ Variable } & \multicolumn{3}{|c|}{ Univariate analysis } & \multicolumn{3}{|c|}{ Multivariate analysis } \\
\hline & HR & $95 \% \mathrm{CI}$ & P-value & HR & $95 \% \mathrm{CI}$ & P-value \\
\hline Age (>70 vs. $\leq 70$ years) & 1.4 & $0.62-3.2$ & 0.42 & - & - & - \\
\hline ECOG-PS score (0 vs. 1) & 0.88 & $0.4-2.1$ & 0.79 & - & - & - \\
\hline Sex (female vs. male) & 0.46 & $0.22-0.95$ & 0.04 & 0.27 & $0.04-1.64$ & 0.16 \\
\hline Histology (non-Sq vs. Sq) & 0.24 & $0.07-0.80$ & 0.02 & 0.44 & $0.15-1.2$ & 0.12 \\
\hline $\begin{array}{l}\text { EGFR mutation status } \\
\text { (positive vs. negative or unknown) }\end{array}$ & 0.41 & $0.20-0.86$ & 0.02 & 0.44 & $0.18-1.1$ & 0.08 \\
\hline $\begin{array}{l}\text { Smoking history } \\
\text { (never smoker vs. former or current smoker) }\end{array}$ & 0.37 & $0.20-0.80$ & $<0.01$ & 0.13 & $0.20-0.85$ & 0.03 \\
\hline $\begin{array}{l}\text { The presence of ZOL-related fever } \\
\text { (yes vs. no) }\end{array}$ & 0.46 & $0.22-0.97$ & 0.04 & 0.37 & $0.16-0.83$ & 0.02 \\
\hline
\end{tabular}

CI, confidence interval; ECOG-PS, Eastern Cooperative Oncology Group-performance status; EGFR, epidermal growth factor receptor; HR, hazard ratio; Sq, squamous cell carcinomas; ZOL, zoledronate.

history [HR (95\% CI) $0.13(0.20-0.85), \mathrm{P}=0.03$ ] were significant independent prognostic factors (Table II). The presence of EGFR mutation tended to be associated with better OS [HR (95\% CI) $0.44(0.15-1.2), P=0.12]$, although this finding was not statistically significant.

\section{Discussion}

In the present study, we compared the characteristics of patients in the fever group with those of patients in the non-fever group in order to identify predictors of ZOL-related fever. Reid et al 
reported that young age and female sex are predictors of the development of fever after ZOL administration in patients with osteoporosis (11). In our study, although patients in the fever group tended to be younger than those in the non-fever group, with a greater percentage of women, these differences were not significant. Further large-scale studies are necessary to confirm whether such characteristics are predictors of ZOL-related fever in patients with cancer.

We compared the OS between the fever group and non-fever group in NSCLC patients treated with ZOL. Although there were no significant differences in patient characteristics between the two groups, the OS in the fever group was significantly longer than that in the non-fever group. This indicates that ZOL-related fever is an important prognostic indicator in advanced or recurrent NSCLC patients with bone metastases. To the best of our knowledge, this is the first report of an association between ZOL-related fever and clinical outcome in patients with cancer. As the time to the first SRE was not significantly different between the two groups, the difference in OS between the two groups is likely not attributable to the preventive effect of ZOL on SREs.

In addition to ZOL-related fever, a positive EGFR mutation status, non-Sq histology, no smoking history, and female sex were significantly associated with longer OS. These characteristics are known prognostic factors in patients with NSCLC, and are strongly associated with the presence of EGFR mutation (12-14). Owing to the emergence of EGFR tyrosine kinase inhibitors, the survival of NSCLC patients with EGFR mutation is better than that of patients without EGFR mutation (13). We performed multivariate analyses to exclude confounding influences, and the results revealed that ZOL-related fever is independently associated with better OS in advanced NSCLC patients with bone metastases.

Two hypotheses may explain the association between ZOL-related fever and improved OS. First, patients in the fever group may have superior immunocompetence than those in the non-fever group, resulting in better prognosis, and ZOL administration results in the development of fever depending on the immunocompetence of the patient. In other words, ZOL-related fever is a prognostic factor regardless of any beneficial clinical effects of ZOL. Second, patients in the fever group alone may benefit from favorable effects of ZOL other than SRE inhibition. Thus, ZOL-related fever is a predictive factor for an undefined, survival-promoting effect of ZOL.

ZOL-related fever is associated with the activation of $\gamma \delta \mathrm{T}$ cells, which recognize non-peptide antigens in a major histocompatibility complex-unrestricted manner (15). $\gamma \delta \mathrm{T}$ cells have demonstrated cytotoxicity against a variety of cell lines in vitro, including peripheral blood mononuclear cells from patients with NSCLC (16). Immunotherapies utilizing $\gamma \delta \mathrm{T}$ cells are currently under development and have been shown to be safe and feasible (17-19). Such therapies could offer invaluable alternatives for cancer treatment in the future. Thus, it is possible that ZOL-related fever is a predictive factor for an additional survival-improving effect of ZOL, and that $\gamma \delta \mathrm{T}$ cells were activated to a greater extent by ZOL in the fever group, resulting in the activation of cancer immunity and improved OS. However, further study is necessary to clarify the mechanism by which ZOL prolongs OS and to confirm whether $\gamma \delta$ T cells are activated after ZOL administration in the fever group. In this regard, we are conducting a prospective observational study to confirm the reproducibility of the correlation between ZOL-related fever and OS and to clarify the precise mechanism of ZOL-related fever development in patients with advanced NSCLC.

In conclusion, the development of ZOL-related fever was independently correlated with better OS in advanced or recurrent NSCLC patients with bone metastases. ZOL-related fever is a predictive factor for an undefined, survival-promoting effect of ZOL.

\section{Acknowledgements}

We would like to thank Editage for English language editing.

\section{References}

1. Siegel R, Naishadham D and Jemal A: Cancer statistics, 2012. CA Cancer J Clin 62: 10-29, 2012.

2. Coleman RE: Metastatic bone disease : Clinical features, pathophysiology and treatment strategies. Cancer Treat Rev 27: 165-176, 2001.

3. Dong M, Feng FY, Zhang Y, Xie GR, Wang YJ, Liu JW, Song ST, Zhou QH, Ren J, Jiao SC, et al: Phase III clinical study of zoledronic acid in the treatment of pain induced by bone metastasis from solid tumor or multiple myeloma. Zhonghua Zhong Liu Za Zhi 30: 215-220, 2008 (In Chinese).

4. Rosen LS, Gordon D, Tchekmedyian S, Yanagihara R, Hirsh V, Krzakowski M, Pawlicki M, de Souza P, Zheng M, Urbanowitz G, et al: Zoledronic acid versus placebo in the treatment of skeletal metastases in patients with lung cancer and other solid tumors: A phase III, double-blind, randomized trial-the Zoledronic Acid Lung Cancer and Other Solid Tumors Study Group. J Clin Oncol 21: 3150-3157, 2003.

5. Kunzmann V, Bauer E, Feurle J, Weissinger F, Tony H and Wilhelm M: Stimulation of gammadelta T cells by aminobisphosphonates and induction of antiplasma cell activity in multiple myeloma. Blood 96: 384-392, 2000.

6. Gnant M, Mlineritsch B, Stoeger H, Luschin-Ebengreuth G, Heck D, Menzel C, Jakesz R, Seifert M, Hubalek M, Pristauz G, et al: Adjuvant endocrine therapy plus zoledronic acid in premenopausal women with early-stage breast cancer: 62-month follow-up from the ABCSG-12 randomised trial. Lancet Oncol 12: 631-641, 2011.

7. Avilés A, Nambo MJ, Neri N, Castañeda C, Cleto S and Huerta-Guzmán J: Antitumor effect of zoledronic acid in previously untreated patients with multiple myeloma. Med Oncol 24: 227-230, 2007.

8. Kunzmann V, Bauer E and Wilhelm M: Gamma/delta T-cell stimulation by pamidronate. N Engl J Med 340: 737-738, 1999.

9. Dicuonzo G, Vincenzi B, Santini D, Avvisati G, Rocci L, Battistoni F, Gavasci M, Borzomati D, Coppola R and Tonini G: Fever after zoledronic acid administration is due to increase in TNF-alpha and IL-6. J Interferon Cytokine Res 23: 649-654, 2003.

10. Bonneville $\mathrm{M}$ and Scotet E: Human Vgamma9Vdelta2 T cells: Promising new leads for immunotherapy of infections and tumors. Curr Opin Immunol 18: 539-546, 2006.

11. Reid IR, Gamble GD, Mesenbrink P, Lakatos P and Black DM: Characterization of and risk factors for the acute-phase response after zoledronic acid. J Clin Endocrinol Metab 95: 4380-4387,2010.

12. Nakamura H, Ando K, Shinmyo T, Morita K, Mochizuki A, Kurimoto $\mathrm{N}$ and Tatsunami S: Female gender is an independent prognostic factor in non-small-cell lung cancer: A meta-analysis. Ann Thorac Cardiovasc Surg 17: 469-480, 2011.

13. Kris MG, Johnson BE, Berry LD, et al: NIH Public Access. Select Target Drugs 311: 1998-2006, 2014.

14. Mitsudomi T, Kosaka T, Endoh H, Horio Y, Hida T, Mori S, Hatooka S, Shinoda M, Takahashi T and Yatabe Y: Mutations of the epidermal growth factor receptor gene predict prolonged survival after gefitinib treatment in patients with non-small-cell lung cancer with postoperative recurrence. J Clin Oncol 23: 2513-2520, 2005.

15. Triebel F and Hercend T: Subpopulations of human peripheral T gamma delta lymphocytes. Immunol Today 10: 186-188, 1989. 
16. Kondo M, Sakuta K, Noguchi A, Ariyoshi N, Sato K, Sato S, Sato K, Hosoi A, Nakajima J, Yoshida Y, et al: Zoledronate facilitates large-scale ex vivo expansion of functional gammadelta $\mathrm{T}$ cells from cancer patients for use in adoptive immunotherapy. Cytotherapy 10: 842-856, 2008.

17. Sakamoto M, Nakajima J, Murakawa T, Fukami T, Yoshida Y, Murayama T, Takamoto S, Matsushita $\mathrm{H}$ and Kakimi K: Adoptive immunotherapy for advanced non-small cell lung cancer using zoledronate-expanded $\gamma \delta$ Tcells: A phase I clinical study. J Immunother 34: 202-211, 2011.
18. Kobayashi H, Tanaka Y, Yagi J, Minato N and Tanabe K: Phase I/II study of adoptive transfer of $\gamma \delta$ T cells in combination with zoledronic acid and IL-2 to patients with advanced renal cell carcinoma. Cancer Immunol Immunother 60: 1075-1084, 2011.

19. Kobayashi $\mathrm{H}$ and Tanaka $\mathrm{Y}: \gamma \delta \mathrm{T}$ cell immunotherapy-A review. Pharmaceuticals (Basel) 8: 40-61, 2015 